Revta brasil. Bot., São Paulo, V.20, n.1, p.67-77, jun. 1997

\title{
Fitossociologia de um remanescente de mata mesófila semidecídua urbana, Bosque John Kennedy, Araguari, MG, Brasil
}

\author{
GLEIN MONTEIRO ARAÚJOํ, ANTÔNIO JOSÉ MAIA GUIMARÃES² e JIMI NAOKI NAKAJIMA
}

(recebido em 09/03/95; aceito em 22/10/96)

\begin{abstract}
The phytosociological study of the semideciduous mesophytic forest in the John Kennedy Bosque, Araguari, MG, Brazil). A phytosociological survey of the species of trees was carried out in a semideciduous mesophytic forest in the John Kennedy Bosque (48 $11^{\prime} 19^{\prime \prime} \mathrm{W}$ and $18^{\circ} 38^{\prime} 35^{\prime \prime}$ 'S), Araguari, western Minas Gerais, Brazil. This area is located in the urban perimeter, and has 11.2 ha occupied mostly by forest. One thousand eight hundred and twenty seven (1827) individuals trees were recorded in 1.2 ha, belonging to 46 families, 88 genera and 113 species, and Shannon's diversity index was 3.75 nats/individual. The phytosociological parameter of IVI show that the most important species are Licania apetala, Micrandra elata, Copaifera langsdorffii, Astronium nelsonrosae and Alchornea glandulosa, and the most important families are Chrysobalanaceae, Euphorbiaceae, Caesalpinaceae, Mimosaceae and Anacardiaceae. The occurrence of some species in the John Kennedy Bosque which are known to be gap colonizers (Alchornea glandulosa and Piptocarpha macropoda) or secondary (Didymopanax macrocarpum and Maprounea guianensis) shows the existence of gap areas in this forest, but the comparison between plots of intact forest and gap forest indicates that the specific structure remains unchanged.
\end{abstract}

RESUMO - (Fitossociologia de um remanescente de mata mesófila semidecídua urbana, Bosque John Kennedy, Araguari, MG, Brasil). Um estudo fitossociológico foi realizado no Bosque Municipal John Kennedy, município de Araguari, MG (48 $11^{\prime} 19^{\prime}$ "W e $18^{\circ} 38^{\prime} 35^{\prime \prime}$ S). O bosque, com aproximadamente 11,2 ha, situa-se no perímetro urbano e é formado principalmente por uma mata mesófila semidecídua. No levantamento fitossociológico realizado, amostraram-se, em 1,2 ha, 1827 indivíduos, pertencentes a 46 famílias; 88 gêneros e 113 espécies, com um índice de diversidade de Shannon de H’ =3,75 nats/indivíduo. Os parâmetros fitossociológicos indicam as espécies mais importantes: Licania apetala, Micrandra elata, Copaifera langsdorffi, Astronium nelson-rosae e Alchornea glandulosa, e as famílias mais importantes: Chrysobalanaceae, Euphorbiaceae, Caesalpinaceae, Mimosaceae e Anacardiaceae. A ocorrência de algumas espécies incluidas entre as principais, caracteristicamente, pioneiras (Alchornea glandulosa e Piptocarpha macropoda), ou secundárias (Didymopanax macrocarpum e Maprounea guianensis), pode ser explicada pela existência de algumas áreas de clareiras, no interior dessa mata semidecídua, mas que, aparentemente, ainda não interferiram na estrutura da mata como um todo.

Key words - Urban semideciduous mesophytic forest, phytosociology, gaps

\section{Introdução}

Na região do cerrado do Brasil central, as matas mesófilas semidecíduas ocorrem em forma de manchas, ocupando, geralmente, as encostas dos interflúvios próximas a cursos d'água ou a nascentes, onde são popularmente conhecidas como “capões de mato" (Rizzini 1963, 1979).

A composição florística e a deciduidade dessas matas são variadas, dependendo das condições de umidade, fertilidade e profundidade do solo (Ratter et al. 1978 a,b, Rizzini 1979, Eiten 1990). Quanto aos aspectos fitossociológicos e características do solo, os estudos básicos dessas fitocenoses são importantes e estão concentrados, principalmente, no estado de São Paulo (Cavasan et al. 1984, Meira Neto et al. 1989, Rodrigues et al. 1989, Martins 1991), mas são raros no

1. Departamnento de Biociências, Universidade Federal de Uberlândia, Caixa Postal 593, 38400-902 Uberlândia, MG, Brasil.

2. Curso de Agronomia, Universidade Federal de Uberlândia, MG.
Brasil central, principalmente no Triângulo Mineiro (Araújo 1992).

Nas regiões mais devastadas, por causa das atividades agrícolas, reflorestamentos e carvoarias, as matas mesófilas restringem-se a pequenas reservas, próximas a nascentes ou a cursos d'água. A maioria, porém, não passa de depósito vivo de madeira. Além disso, remanescentes dessas comunidades vegetais também podem ser encontrados em áreas urbanas. Um exemplo é o Bosque John Kennedy no município de Araguari, Minas Gerais.

Nesse sentido, essas matas podem ser consideradas não só um excelente local para o turismo ecológico, como também uma forma de manutenção do patrimônio fitogenético nativo, além de servir como abrigo para pássaros e outros pequenos animais.

O presente trabalho teve como objetivo determinar a estrutura fitossociológica das espécies arbóreas e as características do solo daquela mata urbana em Araguari. 


\section{Material e métodos}

O Bosque John Kennedy, situado no perímetro urbano do município de Araguari, Triângulo Mineiro (48 11'19'"W e $18^{\circ} 38^{\prime} 35^{\prime \prime}$ 'S), está sendo administrado pela prefeitura da cidade, desde a emancipação do município em 1888. Em sua maior parte, a área de 11,2 ha é ocupada, por uma mata mesófila semidecídua, com árvores de até $25 \mathrm{~m}$, e dossel bastante fechado. Recentemente, a morte natural de várias espécies arbóreas e arbustivas, ocasionou, no interior da mata, algumas clareiras, com mais de $200 \mathrm{~m}^{2}$ de área. Atualmente, essas clareiras, encontram-se em adiantado estádio de recuperação e caracterizam-se pela presença de árvores de pequeno porte ( 3 a $8 \mathrm{~m}$ ), lianas, arbustos e herbáceas. No interior do bosque, utilizado como área de lazer, encontram-se algumas trilhas e construções.

Para a determinação das características físico-químicas, foram coletadas onze amostras compostas de solo, em áreas não perturbadas, numa profundidade de $0-10 \mathrm{~cm}$, por se tratar do horizonte de maior fertilidade nas matas decíduas regionais (Araújo 1992). As análises, quanto aos cátions trocáveis (P, K, $\mathrm{Ca}, \mathrm{Mg}, \mathrm{Al}$ e H), pH, matéria orgânica e textura, foram realizadas no laboratório de solo do Departamento de Agronomia da Universidade Federal de Uberlândia, MG, tendo sido aplicados os métodos convencionais (EMBRAPA 1979).

O levantamento fitossociológico foi realizado, utilizando-se o método de parcelas (Greig-Smith 1983), - 60 parcelas de $20 \mathrm{~m}$ $\mathrm{x} 10 \mathrm{~m}$ - sistematicamente distribuídas na área ocupada pela mata. Doze das parcelas ocupavam ambientes de clareiras. Para a localização das áreas amostradas, em um dos vértices de cada parcela foi implantado um marco de cimento. De cada uma das parcelas foram amostrados todos os indivíduos com $10 \mathrm{~cm}$ ou mais de circunferência de tronco, à altura do peito $(1,30 \mathrm{~m})$ e $2 \mathrm{~m}$ ou mais de altura.

Durante um ano, toda a área estudada foi percorrida periodicamente, para a coleta de exemplares férteis. A identificação do material coletado foi feita com o apoio de literatura especializada, comparações com coleções de herbários, ou por determinações de especialistas. Essa coleção encontra-se depositada no Herbarium Uberlandensis (HUFU) da Universidade Federal de Uberlândia, MG.

Os parâmetros fitossociológicos, e o índice de diversidade de Shannon (H') para espécies, foram determinados, aplicando-se o programa FITOPAC, de autoria do Prof. Dr. G. J. Shepherd do Departamento de Botânica da Universidade Estadual de Campinas. O índice de Sorensen (Mueller-Dombois \& Ellemberg 1974) foi utilizado para calcular a similaridade específica, entre o Bosque John Kennedy e outras matas da região.

Para a classificação sucessional das 10 espécies principais que ocorreram nas clareiras, foram utilizadas informações bibliográficas (Denslow 1980, 1987, Gandolfi et al. 1995) e observações de campo sobre o hábito de vida e local de ocorrência das espécies.

\section{Resultados e Discussão}

O solo sob a mata mesófila semidecídua do bosque de Araguari enquadrou-se na classe de Latossolo Vermelho Escuro (EMBRAPA 1982) e apresentou textura franco argilosa. Os valores de $\mathrm{pH}$ em água $(4,2$ a 4,6) permitiram classificá-lo como de acidez elevada (CFSMG 1989). Esses baixos valores de $\mathrm{pH}$ estão relacionados com a baixa disponibilidade de $\mathrm{K}$, $\mathrm{Ca}$ e $\mathrm{Mg}$, e com os elevados teores de $\mathrm{Al}$ trocável (tabela 1). Quanto à saturação de bases, ocorreram variações percentuais não significativas, uma vez que valores até $25 \%$ são

Tabela 1. Características químicas e físicas da camada superficial $(0-10 \mathrm{~cm})$ do solo sob a mata mesófila do bosque de Araguari, Minas Gerais. $\mathrm{V}=$ bases, $\mathrm{MO}=$ matéria orgânica, $\mathrm{Ar}=$ areia, $\mathrm{S}=$ silte, $\mathrm{A}=$ Argila.

\begin{tabular}{|c|c|c|c|c|c|c|c|c|c|c|c|c|c|}
\hline \multirow{3}{*}{ Amostra } & \multirow{3}{*}{$\begin{array}{c}\mathrm{pH} \\
\mathrm{H}_{2} \mathrm{O}\end{array}$} & \multicolumn{6}{|c|}{ Cátions trocáveis } & \multicolumn{3}{|c|}{ Saturação } & \multicolumn{3}{|c|}{ Textura } \\
\hline & & $P$ & K & $\mathrm{Ca}$ & $\mathrm{Mg}$ & $\mathrm{Al}$ & $\mathrm{H}$ & V & $\mathrm{Al}$ & MO & $\mathrm{Ar}$ & $\mathrm{S}$ & A \\
\hline & & ppm & \multicolumn{5}{|c|}{ Meq/100g } & \multicolumn{6}{|c|}{$\%$} \\
\hline 1 & 4,3 & 1,8 & 0,2 & 0,4 & 0,2 & 1,3 & 8,5 & 7 & 62 & 7,1 & 41 & 17 & 42 \\
\hline 2 & 4,6 & 3,5 & 0,2 & 1,1 & 0,6 & 1,3 & 7,5 & 17 & 42 & 7,4 & 39 & 13 & 49 \\
\hline 3 & 4,3 & 4,9 & 0,2 & 0,9 & 0,2 & 2,2 & 13,1 & 8 & 63 & 8,7 & 39 & 13 & 47 \\
\hline 4 & 4,2 & 2,7 & 0,2 & 0,3 & 0,1 & 2,3 & 8,7 & 5 & 80 & 8,0 & 35 & 18 & 47 \\
\hline 5 & 4,4 & 1,9 & 0,1 & 0,3 & 0,2 & 1,5 & 9,5 & 5 & 73 & 6,5 & 38 & 24 & 38 \\
\hline 6 & 4,3 & 2,9 & 0,2 & 0,3 & 0,3 & 1,4 & 9,6 & 7 & 64 & 7,4 & 38 & 22 & 40 \\
\hline 7 & 4,0 & 3,9 & 0,2 & 0,2 & 0,2 & 1,0 & 16,1 & 3 & 66 & 9,5 & 41 & 16 & 43 \\
\hline 8 & 4,2 & 2,2 & 0,2 & 0,2 & 0,2 & 2,2 & 13,1 & 3 & 82 & 9,2 & 47 & 22 & 32 \\
\hline 9 & 4,3 & 3,3 & 0,1 & 0,4 & 0,2 & 1,8 & 7,0 & 7 & 71 & 7,4 & 37 & 27 & 36 \\
\hline 10 & 4,2 & 2,3 & 0,2 & 0,2 & 0,1 & 2,0 & 7,8 & 4 & 84 & 7,4 & 45 & 22 & 34 \\
\hline 11 & 4,2 & 3,1 & 0,2 & 0,2 & 0,1 & 2,1 & 10,2 & 4 & 80 & 8,0 & 45 & 23 & 33 \\
\hline Média & 4,3 & 3,0 & 0,2 & 0,4 & 0,2 & 1,7 & 10,1 & 6 & 70 & 7,9 & 40 & 20 & 40 \\
\hline D.padrão & 0,1 & 0,9 & 0,1 & 0,3 & 0,1 & 0,5 & 2,8 & 4 & 12 & 0,9 & 4 & 4,6 & 6 \\
\hline
\end{tabular}


considerados muito baixos (CFSMG 1989). Solos distróficos como esse são comumente encontrados sob algumas matas mesófilas semidecíduas no Triângulo Mineiro (Araújo 1992).

No levantamento fitossociológico realizado, em 1,2 ha da mata, foram amostradas 1827 árvores com área basal média de $39,47 \mathrm{~m}^{2}$. Os indivíduos representaram 113 espécies distribuídas em 88 gêneros e 46 famílias. Os gêneros com maior número de espécies foram: Machaerium e Myrcia com 4, Aspidosperma, Casearia, Inga e Ocotea, com 3 espécies cada um (tabela 2).

A similaridade entre as espécies da comunidade vegetal do presente estudo, e de outras matas mesófilas próximas foi de $71 \%$, com a mata mesófila semidecídua da Fazenda Experimental do Glória, e

Tabela 2. Espécies arbóreas amostradas no levantamento fitossociológico do bosque de Araguari, Minas Gerais e sua ocorrência em outras matas do Triângulo Mineiro: matas mesófilas semidecíduas da Fazenda Experimental do Glória (G) e da Estação Ecológica do Panga (P) (Araújo 1992) e mata galeria da Estação Ecológica do Panga (GP) (Schiavini 1992). * = ocorrência.

\begin{tabular}{|c|c|c|c|c|}
\hline $\begin{array}{l}\text { Família } \\
\text { Espécie }\end{array}$ & Nome popular & G & $\mathrm{P}$ & GP \\
\hline \multicolumn{5}{|l|}{ ANACARDIACEAE } \\
\hline Astronium nelson- rosae D.A. Santin & Guaritá & $*$ & & \\
\hline Tapirira peckoltiana Engl. & Pau-pombo & $*$ & & \\
\hline \multicolumn{5}{|l|}{ ANNONACEAE } \\
\hline Annona cacans Warm. & Araticum-cagão & $*$ & & \\
\hline Cardiopetalum calophyllum Schl. & Pimenta-de-macaco & * & * & \\
\hline Duguetia lanceolata St. Hil. & Pindaiva & $*$ & * & \\
\hline Unonopsis lindmanis R.E.Fa. & Araticum & & & \\
\hline Xylopia aromatica Lam. & Pindaiba & $*$ & * & \\
\hline Xylopia sericea St. Hil. & Pimenta-de-macaco & $*$ & & \\
\hline \multicolumn{5}{|l|}{ APOCYNACEAE } \\
\hline Aspidosperma cylindrocarpum Muell. Arg. & Peroba & $*$ & * & * \\
\hline Aspidosperma parvifolium A. DC. & Tambú & $*$ & * & \\
\hline Aspidosperma discolor A. DC. & Canela-de-velha & $*$ & & \\
\hline \multicolumn{5}{|l|}{ ARALIACEAE } \\
\hline Didymopanax morototoni Dene \& Planch. & Mandiocão & $*$ & & \\
\hline \multicolumn{5}{|l|}{ ASTERACEAE } \\
\hline Piptocarpha macropoda Baker. & Coração-de-negro & $*$ & & \\
\hline \multicolumn{5}{|l|}{ BIGNONIACEAE } \\
\hline Jacaranda macrantha Cham. & Castanhola & * & & \\
\hline Tabebuia serratifolia (Vahl.) Nichols. & Ipê-amarelo & $*$ & & \\
\hline Tabebuia sp. & Ipê & & & \\
\hline \multicolumn{5}{|l|}{ BOMBACACEAE } \\
\hline Eriotheca candolleana (K.Schum.) A.Robyns & Paininha & $*$ & $*$ & \\
\hline Chorisia speciosa St. Hil. & Barriguda & & & \\
\hline \multicolumn{5}{|l|}{ BORAGINACEAE } \\
\hline Cordia sellowiana Cham. & Chá-de-bugre & $*$ & & \\
\hline \multicolumn{5}{|l|}{ BURSERACEAE } \\
\hline Protium heptaphyllum (Aubl.) March. & Amescla & $*$ & $*$ & $*$ \\
\hline \multicolumn{5}{|l|}{ CAESALPINACEAE } \\
\hline Apuleia leiocarpa (Vog.) Macbr. & Garapa & $*$ & & \\
\hline Bauhinia sp. & Unha-de-vaca & & & \\
\hline Cassia ferruginea Schrad. ex DC. & Canafista & $*$ & & \\
\hline Cassia sp. & Amarelinho & & & \\
\hline Copaifera langsdorffii Desf. & Pau-de-óleo & $*$ & * & * \\
\hline Hymenaea courbaril $\mathrm{L}$. & Jatobá & $*$ & $*$ & * \\
\hline Sclerolobium paniculatum Vog. & Carvoeiro & & & \\
\hline Swartzia apetala Raddi & Grão-de-galo & & & \\
\hline \multicolumn{5}{|l|}{ CECROPIACEAE } \\
\hline Cecropia pachystachya Trec. & Embaúba & & & * \\
\hline \multicolumn{5}{|l|}{ CELASTRACEAE } \\
\hline Maytenus sp1. & Congonha & & & \\
\hline Maytenus sp2. & Cafezinho & & & \\
\hline \multicolumn{5}{|l|}{ CHRYSOBALANACEAE } \\
\hline Hirtella glandulosa Spreng. & Tento & & & \\
\hline
\end{tabular}


(cont.)

Família

Espécie

Nome popular

G $\quad P \quad$ GP

Hirtella gracilipes (Hook. f.) Prance

Licania apetala (E. Mey) Fritsch.

CLUSIACEAE

Rheedia gardneriana Planch. \& Triana

Pau-de-espeto

Rapadura

COMBRETACEAE

Terminalia brasiliensis Eichl.

ELAEOCARPACEAE

Sloanea monosperma Vell.

EUPHORBIACEAE

Alchornea glandulosa Popp.

Maprounea guianensis Aubl.

Micrandra elata Mull. Arg.

Pera glabrata (Schott.) Baill.

FABACEAE

Machaerium nictitans (Vell.) Benth.

Machaerium sp.

Machaerium stipitatum (DC.) Vog.

Machaerium villosum Vog.

Ormosia arborea (Vell.) Harms.

Platypodium elegans Vog.

Sweetia fruticosa Spreng.

FLACOURTIACEAE

Casearia grandiflora Camb.

Casearia sylvestris $\mathrm{Sw}$.

Casearia gossypiosperma Briquet

HIPPOCRATEACEAE

Cheiloclinium cognatum (Miers) A.C.Smith

LACISTEMATACEAE

Lacistema aggregatum (Berg.) Rusby

LAURACEAE

Cryptocarya aschersoniana $\mathrm{Mez}$

Nectandra cissiflora Nees

Ocotea corymbosa (Meissn.) Mez

Ocotea spixiana (Nees) Mez

Ocotea sp.

LECYTHIDACEAE

Cariniana estrellensis (Raddi) O. Ktze.

MELASTOMATACEAE

Miconia sellowiana Naud.

Miconia sp.

Mouriri apiranga Spruce \& Triana

MELIACEAE

Cabralea cangerana (Vell. ) Mart.

Cedrela fissilis Vell.

Trichilia pallida $\mathrm{Sw}$.

MIMOSACEAE

Albizia polycephala (Benth. ) Killip

Inga fagifolia Willd.

Inga $\mathrm{sp} .1$

Inga sp. 2

MONIMIACEAE

Siparuna guianensis Aubl.

Siparuna sp.

MORACEAE

Ficus enormis (Mart.\& Miq.) Miguel.

Ficus cf. trigonata L. F.

Maclura tinctoria (L.) Don.

Sorocea bomplandii (Baill.) W. Burg.

Bacupari

Capitão-garroti

Coloral-da-mata

Urucurana

Marfim

Mamoneira

Tamanqueira

Bico-de-pato

Jacarandá

Sapuvinha

Jacarandá-paulista

Tento

Jacarandá

Bico-de-pato

Pindaiba-preta

Erva-de-lagarto

Guaçatonga

Bacupari

Cafezinho

Batalheira

Canela-sassafrás

Canelão

Canela-de-cheiro

Canela

Jequitibá

Jacatirão

Olho-de-pombo

Apiranga

Canjerana

Cedro

Catigua

Canafístula

Angá-branco

Angazinho

Angá

Negramínea

Sassafrás

Gameleira

Gameleira

Amoreira

Serralha-da-mata

* *

* $\quad *$

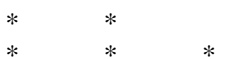

$*$
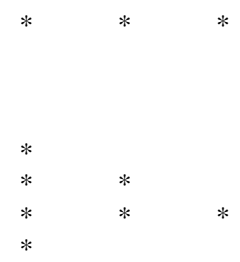

(1)

*

$*$

$*$
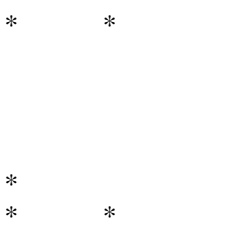
(cont.)

\begin{tabular}{|c|c|c|c|c|}
\hline $\begin{array}{l}\text { Família } \\
\text { Espécie }\end{array}$ & Nome popular & G & $\mathrm{P}$ & GP \\
\hline \multicolumn{5}{|l|}{ MYRISTICACEAE } \\
\hline Virola sebifera Aubl. & Pindaibão & $*$ & $*$ & $*$ \\
\hline \multicolumn{5}{|l|}{ MYRSINACEAE } \\
\hline Rapanea lancifolia (Mart.) Mez. & Pororoquinha & & & \\
\hline Rapanea umbellata (Mart.) Mez. & Pororoca & $*$ & $*$ & $*$ \\
\hline \multicolumn{5}{|l|}{ MYRTACEAE } \\
\hline Calyptranthes clusiaefolia (Miq.) Berg & Pitanga & & & \\
\hline Eugenia florida DC. & Guamirim & $*$ & & \\
\hline Gomidesia brunea Camb. & Guamirim & & & \\
\hline Myrcia rostrata DC. & Cambui & $*$ & $*$ & \\
\hline Myrcia sp.1 & Guamirim & & & \\
\hline Myrcia sp.2 & Guamirim & & & \\
\hline Myrcia tomentosa DC. & Goiabeira-brava & $*$ & $*$ & $*$ \\
\hline Siphoneugena densiflora Berg & Cravinho & $*$ & & \\
\hline \multicolumn{5}{|l|}{ NYCTAGINACEAE } \\
\hline Guapira cacerensis (Hoehne) Lundell & Maria-mole & & & \\
\hline \multicolumn{5}{|l|}{ OLACACEAE } \\
\hline Heisteria ovata Benth. & Umari & $*$ & & \\
\hline \multicolumn{5}{|l|}{ OPILIACEAE } \\
\hline Agonandra brasiliensis Miers & Quina-da-mata & $*$ & $*$ & $*$ \\
\hline \multicolumn{5}{|l|}{ PIPERACEAE } \\
\hline Piper arboreum Aubl. & Jaborandi & & & \\
\hline \multicolumn{5}{|l|}{ PROTEACEAE } \\
\hline Roupala brasiliensis Klotzsch & Carne-de-vaca & $*$ & $*$ & $*$ \\
\hline \multicolumn{5}{|l|}{ ROSACEAE } \\
\hline Prunus sp. & Pessegueiro-bravo & & & \\
\hline \multicolumn{5}{|l|}{ RUBIACEAE } \\
\hline Alibertia sessilis (Vell.) K. Schum & Marmelada & $*$ & $*$ & \\
\hline \multicolumn{5}{|l|}{ Amayoua intermedia cf. var. brasiliana } \\
\hline Steyerm. & Canela-de-veado & $*$ & & \\
\hline Faramea cyanea Müll. Arg. & Cafezinho & $*$ & $*$ & $*$ \\
\hline Ixora warmingii Müll. Arg. & Jasmim-da-mata & $*$ & $*$ & \\
\hline Psychotria sp. & Pau-de-espeto & & & \\
\hline \multicolumn{5}{|l|}{ RUTACEAE } \\
\hline Metrodorea pubescens St. Hil.\& Tul. & Carrapateiro & & & \\
\hline Zanthoxylum rhoifolium Lam. & Laranjeira & $*$ & $*$ & \\
\hline Zanthoxylum riedelianum Engler & Maminha-de-porca & & & \\
\hline \multicolumn{5}{|l|}{ SAPINDACEAE } \\
\hline Cupania vernalis Camb. & Cambuatá & $*$ & $*$ & $*$ \\
\hline Matayba guianensis Aubl. & Cambuatá & $*$ & $*$ & $*$ \\
\hline \multicolumn{5}{|l|}{ SAPOTACEAE } \\
\hline \multicolumn{5}{|l|}{ Micropholis venulosa (Mat. \& Eichler) } \\
\hline Pierre & Pitanga & & & \\
\hline Pouteria sp. & Guapeva & & & \\
\hline \multicolumn{5}{|l|}{ STYRACACEAE } \\
\hline Styrax acuminatum Pohl. & Laranjeira & & & \\
\hline \multicolumn{5}{|l|}{ TILIACEAE } \\
\hline Luehea grandiflora Mart. \& Zucc. & Açoita-cavalo & & & \\
\hline ULMACEAE & & & & \\
\hline Celtis iguanaea (Jacq.) Sargent & Esporão & $*$ & $*$ & \\
\hline VERBENACEAE & & & & \\
\hline Aegiphila sellowiana Cham. & Tamanqueira & $*$ & $*$ & \\
\hline Vitex polygama Cham. & Tarumã & $*$ & & \\
\hline VOCHYSIACEAE & & & & \\
\hline Callisthene major Mart. & João-farinha & $*$ & & \\
\hline Qualea dichotoma (Mart.) Warm. & Cascudo & $*$ & $*$ & $*$ \\
\hline Qualea jundiahy Warm. & Pau-terra & $*$ & $*$ & $*$ \\
\hline Vochysia tucanorum Mart. & Pau-de-tucano & $*$ & $*$ & $*$ \\
\hline
\end{tabular}


de $35 \%$ e $32 \%$ com a mata mesófila e mata galeria da Estação Ecológica do Panga (tabela 2), todas localizadas no município de Uberlândia. A maior semelhança com a mata da Fazenda do Glória deve-se, possivelmente, às mesmas condições fisico-químicas dos solos, ambos Latossolos originários de sedimentos cenozóicos (EMBRAPA 1982, Baccaro 1990), com baixa fertilidade natural. Além das características do solo, outros fatores como: o histórico da ocupação do ambiente, o corte seletivo de madeira, a atual fase sucessional das comunidades e as limitações do tamanho da amostragem, podem ter interferido para que ocorressem menores valores de similaridade entre a fitocenose do presente trabalho e as da Estação Ecológica do Panga.

O índice de diversidade de Shannon para espécies $\left(\mathrm{H}^{\prime}=3,75\right.$ nats/indivíduo) indica que a mata mesófila de Araguari situa-se entre os valores obtidos em outros estudos realizados no mesmo tipo de formação vegetal: $\mathrm{H}^{\prime}=3,56$ a 4,29 nats/indivíduo (Rodrigues 1986, Bertoni \& Martins 1987, Pagano et al. 1987, Cesar 1988, Mathes et al. 1988, Martins 1991, Araújo 1992, Felfili \& Silva JR. 1992). Apesar de ser um remanescente vegetal situado em área urbana, ainda possui uma diversidade específica que se aproxima de muitas outras florestas protegidas.

As famílias Caesalpinaceae e Myrtaceae, com 8 espécies, Fabaceae com 7, Annonaceae, Lauraceae e Rubiaceae com 5 (tabela 2) tiveram o maior número de espécies. Em geral, essas famílias apresentaram significativa riqueza de espécies em trabalhos realizados nas matas do Triângulo Mineiro (Araújo 1992, Schiavini 1992), Sul de Minas Gerais (Oliveira-Filho et al. 1994) e em outras regiões (Leitão-Filho 1986, Meira-Neto et al. 1989, Rodrigues et al. 1989).

Nas famílias Chrysobalanaceae, Euphorbiaceae, Caesalpinaceae, Mimosaceae e Anacardiaceae foram registrados o maior número de indivíduos amostrados, sendo que as duas primeiras apresentaram $34 \%$ do total. As 15 primeiras famílias tiveram $76 \%$ do IVI total e $81 \%$ do IVC (tabela 3 ). O maior IVI da família Chrysobalanaceae deve-se à alta densidade da espécie Licania apetala, enquanto o da família Euphorbiaceae, principalmente à elevada dominância de Micrandra elata. Essas duas espécies predominam tanto no dossel superior como no subosque da mata, o que indica boa adaptação às condições atuais do ambiente. Já as espécies das famílias Caeasalpinaceae e Fabaceae ocupam o dossel superior, enquanto Myrtaceae e Rubiaceae encontram-se no subosque.

As cinco espécies que obtiveram os maiores IVI's (tabela 4) foram: Licania apetala, Micranda elata, Copaifera langsdorffii, Astronium nelson-rosae e Alchornia glandulosa, representando $34 \%$ do total. Dentre as espécies citadas, as quatro primeiras tiveram IVI acima de 12 , ou seja, cada uma delas representou pelo menos $3,7 \%$ do IVI total. A espécie Licania apetala apresentou maiores valores, graças ao grande número de indivíduos encontrados; Micrandra elata e Copaifera langsdorffii pela dominância; Astronium nelson-rosae, pelos três parâmetros que compõem o IVI. Estas três últimas espécies foram amostradas no subosque, mas com o maior número de indivíduos compondo o dossel superior da mata.

As duas espécies mais importantes desse levantamento, Licania apetala e Micrandra elata, não foram encontradas em outros levantamentos florísticos ou fitossociológicos realizados em matas do Triângulo Mineiro (Araújo 1992, Schiavini 1992). Por outro lado, Copaifera langsdorffii foi amostrada com altos valores de importância nesses mesmos estudos, o que indica tratar-se de uma das espécies com ampla distribuição, e dominância nas matas da região. A limitada ocorrência de Astronium nelson-rosae, provavelmente, deve-se ao fato de a mesma ter sido descrita mais recentemente (Santin 1991), portanto, desconhecida para os autores de estudos realizados anteriormente.

As espécies Cecropia pachystachya, Cordia sellowiana, Miconia sellowiana, Myrcia rostrata e Zanthoxylum rhoifolium, segundo (Gandolfi et al. 1995), apresentam características de pioneiras. Essas espécies ocorrem no bosque de Araguari com mais de 5 indivíduos ha-1 (tabela 4) e indicam a existência de algumas clareiras no interior da mata, devido à mortalidade natural de algumas árvores.

A análise comparativa dos parâmetros fitossociológicos das 10 espécies com maior IVI que ocorreram nas 12 parcelas, em áreas de clareiras, e seus respectivos valores nas áreas intactas (figura 1) permite melhor interpretação dos resultados obtidos, para o conjunto de todas as parcelas. Apenas três espécies foram as mais importantes, em termos de 
Tabela 3. Relação das famílias amostradas, em ordem de índice de valor e importância (IVI), na mata mesófila do bosque Araguari, Minas Gerais, e os outros parâmetros fitossociológicos estimados: $\mathrm{n}=$ número de indivíduos amostrados, $\mathrm{DR}=$ densidade relativa $(\%)$, DoR = dominância relativa (\%), FR = frequiência relativa (\%) e IVC = índice de valor de cobertura (\%).

\begin{tabular}{|c|c|c|c|c|c|c|}
\hline Família & $\mathrm{n}$ & DR & DoR & FR & IVI & IVC \\
\hline Chrysobalanaceae & 376 & 20,58 & 15,40 & 7,72 & 43,72 & 35,98 \\
\hline Euphorbiaceae & 246 & 13,46 & 20,39 & 6,83 & 40,69 & 33,86 \\
\hline Caesalpinaceae & 106 & 5,80 & 13,77 & 5,93 & 25,50 & 19,57 \\
\hline Mimosaceae & 73 & 4,00 & 6,64 & 5,03 & 15,66 & 10,63 \\
\hline Anacardiaceae & 80 & 4,38 & 4,99 & 5,93 & 15,30 & 9,37 \\
\hline Lauraceae & 62 & 3,39 & 6,78 & 3,87 & 14,04 & 10,17 \\
\hline Vochysiaceae & 45 & 2,46 & 4,99 & 3,87 & 11,32 & 7,45 \\
\hline Annonaceae & 72 & 3,94 & 2,28 & 4,38 & 10,61 & 6,23 \\
\hline Rubiaceae & 78 & 4,27 & 1,08 & 4,64 & 9,98 & 5,34 \\
\hline Celastraceae & 52 & 2,85 & 1,81 & 3,48 & 8,13 & 4,65 \\
\hline Monimiaceae & 65 & 3,56 & 0,66 & 3,87 & 8,09 & 4,22 \\
\hline Hippocrateaceae & 63 & 3,45 & 0,88 & 3,74 & 8,07 & 4,33 \\
\hline Sapotaceae & 33 & 1,81 & 1,59 & 3,09 & 6,49 & 3,39 \\
\hline Myristicaceae & 29 & 1,59 & 1,71 & 2,19 & 5,49 & 3,30 \\
\hline Myrtaceae & 37 & 2,03 & 0,37 & 2,96 & 5,36 & 2,40 \\
\hline Fabaceae & 26 & 1,42 & 1,64 & 2,06 & 5,12 & 3,06 \\
\hline Asteraceae & 47 & 2,57 & 1,36 & 1,16 & 5,09 & 3,93 \\
\hline Apocynaceae & 23 & 1,26 & 1,50 & 2,32 & 5,08 & 2,76 \\
\hline Olacaceae & 37 & 2,03 & 1,15 & 1,80 & 4,98 & 3,18 \\
\hline Combretaceae & 17 & 0,93 & 0,94 & 1,93 & 3,81 & 1,87 \\
\hline Flacourtiaceae & 28 & 1,53 & 0,20 & 1,93 & 3,67 & 1,74 \\
\hline Araliaceae & 25 & 1,37 & 0,83 & 1,29 & 3,48 & 2,20 \\
\hline Melastomataceae & 25 & 1,37 & 0,35 & 1,68 & 3,39 & 1,72 \\
\hline Verbenaceae & 13 & 0,71 & 0,99 & 1,68 & 3,38 & 1,70 \\
\hline Burceraceae & 17 & 0,93 & 0,87 & 1,42 & 3,22 & 1,80 \\
\hline Bignoniaceae & 12 & 0,66 & 0,95 & 1,42 & 3,03 & 1,61 \\
\hline Meliaceae & 20 & 1,09 & 0,27 & 1,42 & 2,78 & 1,37 \\
\hline Proteaceae & 12 & 0,66 & 0,40 & 1,55 & 2,60 & 1,05 \\
\hline Borraginaceae & 12 & 0,66 & 0,43 & 1,42 & 2,51 & 1,09 \\
\hline Bombacaceae & 8 & 0,44 & 0,83 & 0,90 & 2,17 & 1,27 \\
\hline Moraceae & 7 & 0,38 & 0,95 & 0,82 & 2,16 & 1,34 \\
\hline Rutaceae & 10 & 0,55 & 0,45 & 0,90 & 1,90 & 1,00 \\
\hline Elaeocarpaceae & 8 & 0,44 & 0,52 & 0,90 & 1,86 & 0,96 \\
\hline Cecropiaceae & 10 & 0,55 & 0,32 & 0,86 & 1,72 & 0,86 \\
\hline Sapindaceae & 7 & 0,38 & 0,38 & 0,90 & 1,67 & 0,76 \\
\hline Lacistemataceae & 10 & 0,55 & 0,04 & 0,90 & 1,49 & 0,58 \\
\hline Lecythidaceae & 4 & 0,22 & 0,68 & 0,39 & 1,28 & 0,89 \\
\hline Myrcinaceae & 7 & 0,38 & 0,09 & 0,64 & 1,11 & 0,47 \\
\hline Styracaceae & 7 & 0,38 & 0,03 & 0,52 & 0,93 & 0,41 \\
\hline Tiliaceae & 5 & 0,27 & 0,08 & 0,52 & 0,87 & 0,36 \\
\hline Clusiaceae & 4 & 0,22 & 0,08 & 0,39 & 0,69 & 0,30 \\
\hline Opiliaceae & 2 & 0,11 & 0,20 & 0,13 & 0,44 & 0,31 \\
\hline Nyctaginaceae & 2 & 0,11 & 0,04 & 0,26 & 0,41 & 0,15 \\
\hline Piperaceae & 3 & 0,16 & 0,01 & 0,13 & 0,30 & 0,17 \\
\hline Rosaceae & 1 & 0,05 & 0,05 & 0,13 & 0,23 & 0,10 \\
\hline Ulmaceae & 1 & 0,05 & 0,03 & 0,13 & 0,21 & 0,09 \\
\hline
\end{tabular}


Tabela 4. Relação das espécies, em ordem de índice de valor de importância (IVI), amostradas na mata mesófila do bosque de Araguari, Minas Gerais, e os outros parâmetros fitossociológicos estimados: $\mathrm{n}=$ número de indivíduos, $\mathrm{DR}=$ densidade relativa $(\%)$, DoR $=$ dominância relativa $(\%), \mathrm{FR}=$ frequiência relativa $(\%)$ e IVC = índice de valor de cobertura $(\%)$.

\begin{tabular}{|c|c|c|c|c|c|c|}
\hline Espécie & $\mathrm{n}$ & DR & DoR & FR & IVI & IVC \\
\hline 1. Licania apetala & 383 & 18,46 & 13,82 & 6,10 & 38,37 & 32,27 \\
\hline 2. Micrandra elata & 123 & 6,74 & 16,28 & 4,06 & 27,08 & 23,02 \\
\hline 3. Copaifera langsdorffii & 51 & 2,79 & 10,04 & 3,42 & 16,26 & 12,83 \\
\hline 4. Astronium nelson-rosae & 66 & 3,61 & 3,09 & 4,49 & 11,19 & 6,70 \\
\hline 5. Alchornea glandulosa & 100 & 5,48 & 1,23 & 1,93 & 8,63 & 6,71 \\
\hline 6. Inga fagifolia & 21 & 1,15 & 4,95 & 1,82 & 7,92 & 6,10 \\
\hline 7. Duguetia lanceolata & 57 & 3,12 & 1,39 & 3,21 & 7,72 & 4,52 \\
\hline 8. Cheiloclinium cognatum & 63 & 3,45 & 0,88 & 3,10 & 7,43 & 4,33 \\
\hline 9. Ocotea spixiana & 34 & 1,86 & 3,37 & 2,03 & 7,27 & 5,24 \\
\hline 10. Maytenus sp.1 & 49 & 2,68 & 1,80 & 2,78 & 7,26 & 4,48 \\
\hline 11. Qualea jundiahy & 32 & 1,75 & 2,18 & 2,46 & 6,40 & 3,94 \\
\hline 12. Inga sp.1 & 45 & 2,46 & 0,90 & 2,89 & 6,25 & 3,36 \\
\hline 13. Maprounea guianensis & 21 & 1,15 & 2,85 & 1,93 & 5,93 & 4,00 \\
\hline 14. Siparuna guianensis & 55 & 3,01 & 0,32 & 2,57 & 5,90 & 3,34 \\
\hline 15. Amayoua intermedia & 41 & 2,25 & 0,31 & 2,67 & 5,23 & 2,55 \\
\hline 16. Virola sebifera & 29 & 1,59 & 1,71 & 1,82 & 5,12 & 3,30 \\
\hline 17. Micropholis venulosa & 27 & 1,48 & 1,49 & 2,14 & 5,11 & 2,97 \\
\hline 18. Piptocarpha macropoda & 47 & 2,57 & 1,36 & 0,96 & 4,90 & 3,94 \\
\hline 19. Heisteria ovata & 37 & 2,03 & 1,15 & 1,50 & 4,68 & 3,18 \\
\hline 20. Tapirira peckoltiana & 14 & 0,77 & 1,91 & 1,50 & 4,17 & 2,67 \\
\hline 21. Hymenaea courbaril & 14 & 0,77 & 1,77 & 1,50 & 4,03 & 2,53 \\
\hline 22. Hirtella glandulosa & 24 & 1,31 & 1,12 & 1,39 & 3,82 & 2,43 \\
\hline 23. Apulea leiocarpa & 16 & 0,88 & 1,64 & 1,28 & 3,80 & 2,52 \\
\hline 24. Teminanalia brasiliensis & 17 & 0,93 & 0,95 & 1,60 & 3,48 & 1,88 \\
\hline 25. Callisthene major & 8 & 0,44 & 2,30 & 0,64 & 3,37 & 2,73 \\
\hline 26. Didymopanax morototoni & 25 & 1,37 & 0,83 & 1,07 & 3,27 & 2,20 \\
\hline 27. Aspidosperma discolor & 17 & 0,93 & 0,71 & 1,60 & 3,24 & 1,64 \\
\hline 28. Ocotea sp. & 11 & 0,60 & 1,37 & 1,07 & 3,04 & 1,97 \\
\hline 29. Protium heptaphyllum & 17 & 0,93 & 0,87 & 1,18 & 2,98 & 1,80 \\
\hline 30. Ocotea corymbosa & 11 & 0,60 & 1,62 & 0,75 & 2,97 & 2,22 \\
\hline 31. Ixora warmingii & 16 & 0,88 & 0,59 & 1,18 & 2,65 & 1,47 \\
\hline 32. Hirtella gracilipes & 14 & 0,77 & 0,47 & 1,28 & 2,52 & 1,23 \\
\hline 33. Casearia grandiflora & 22 & 1,20 & 0,10 & 1,18 & 2,48 & 1,31 \\
\hline 34. Roupala brasiliensis & 12 & 0,66 & 0,40 & 1,28 & 2,34 & 1,05 \\
\hline 35. Cordia sellowiana & 12 & 0,66 & 0,43 & 1,18 & 2,27 & 1,09 \\
\hline 36. Miconia sellowiana & 15 & 0,82 & 0,12 & 1,07 & 2,01 & 0,94 \\
\hline 37. Myrcia rostrata & 15 & 0,82 & 0,07 & 1,07 & 1,96 & 0,89 \\
\hline 38. Cabralea cangerana & 13 & 0,71 & 0,22 & 0,86 & 1,79 & 0,94 \\
\hline 39. Vitex polygama & 6 & 0,33 & 0,80 & 0,64 & 1,77 & 1,13 \\
\hline 40. Siparuna sp. & 10 & 0,55 & 0,34 & 0,86 & 1,75 & 0,89 \\
\hline 41. Cecropia pachystachya & 10 & 0,55 & 0,32 & 0,86 & 1,72 & 0,86 \\
\hline 42. Sloanea monospema & 8 & 0,44 & 0,52 & 0,75 & 1,71 & 0,96 \\
\hline 43. Albizia polycephala & 6 & 0,33 & 0,78 & 0,43 & 1,54 & 1,11 \\
\hline 44. Alibertia sessilis & 9 & 0,55 & 0,08 & 0,86 & 1,48 & 0,63 \\
\hline 45. Faramea cyanea & 11 & 0,60 & 0,11 & 0,75 & 1,46 & 0,71 \\
\hline 46. Siphoneugenia densiflora & 11 & 0,44 & 0,21 & 0,75 & 1,40 & 0,65 \\
\hline 47. Zanthoxylum rhoifolium & 7 & 0,38 & 0,36 & 0,64 & 1,39 & 0,75 \\
\hline 48. Lacistema aggregatum & 10 & 0,55 & 0,04 & 0,75 & 1,33 & 0,58 \\
\hline 49. Aegiphila sellowiana & 7 & 0,38 & 0,19 & 0,75 & 1,32 & 0,57 \\
\hline 50. Chorisia speciosa & 5 & 0,27 & 0,57 & 0,43 & 1,28 & 0,85 \\
\hline 51. Annona cacans & 3 & 0,16 & 0,76 & 0,32 & 1,24 & 0,92 \\
\hline 52. Cariniana estrellensis & 4 & 0,22 & 0,68 & 0,32 & 1,22 & 0,89 \\
\hline 53. Ficus enormis & 3 & 0,16 & 0,72 & 0,32 & 1,20 & 0,88 \\
\hline 54. Swartizia apetala & 12 & 0,66 & 0,11 & 0,43 & 1,19 & 0,77 \\
\hline 55. Sweetia fruticosa & 3 & 0,16 & 0,69 & 0,32 & 1,18 & 0,86 \\
\hline
\end{tabular}


Revta brasil. Bot., São Paulo, 20(1):67-77, jun. 1997

(cont.)

\begin{tabular}{|c|c|c|c|c|c|c|}
\hline Espécie & $\mathrm{n}$ & DR & DoR & FR & IVI & IVC \\
\hline 56. Platypodium elegans & 9 & 0,49 & 0,25 & 0,43 & 1,17 & 0,74 \\
\hline 57. Jacaranda macrantha & 5 & 0,27 & 0,32 & 0,53 & 1,13 & 0,60 \\
\hline 58. Pouteria sp. & 6 & 0,33 & 0,11 & 0,64 & 1,08 & 0,44 \\
\hline 59 Tabebuia sp. & 3 & 0,16 & 0,51 & 0,32 & 1,00 & 0,68 \\
\hline 60 Machaerium villosum & 3 & 0,16 & 0,51 & 0,32 & 1,00 & 0,68 \\
\hline 61. Qualea dichotoma & 4 & 0,22 & 0,34 & 0,43 & 0,99 & 0,56 \\
\hline 62. Sclerolobium paniculatum & 5 & 0,27 & 0,10 & 0,53 & 0,91 & 0,37 \\
\hline 63. Xylopia sericea & 6 & 0,33 & 0,04 & 0,53 & 0,90 & 0,37 \\
\hline 64. Cryptocarya aschersoniana & 4 & 0,22 & 0,21 & 0,43 & 0,86 & 0,43 \\
\hline 65. Matayba guianensis & 4 & 0,22 & 0,21 & 0,43 & 0,86 & 0,43 \\
\hline 66. Styrax acuminatum & 7 & 0,38 & 0,03 & 0,43 & 0,84 & 0,41 \\
\hline 67. Miconia sp. & 8 & 0,44 & 0,07 & 0,32 & 0,83 & 0,51 \\
\hline 68. Rapanea lancifolia & 6 & 0,33 & 0,06 & 0,43 & 0,82 & 0,39 \\
\hline 69. Luehea grandiflora & 5 & 0,27 & 0,08 & 0,43 & 0,78 & 0,36 \\
\hline 70. Tabebuia serratifolia & 4 & 0,22 & 0,11 & 0,43 & 0,76 & 0,33 \\
\hline 71. Trichilia pallida & 5 & 0,27 & 0,04 & 0,43 & 0,75 & 0,32 \\
\hline 72. Eriotheca candolleana & 3 & 0,16 & 0,26 & 0,32 & 0,74 & 0,42 \\
\hline 73. Aspidosperma parvifolium & 3 & 0,16 & 0,47 & 0,11 & 0,74 & 0,64 \\
\hline 74. Casearia sylvestris & 5 & 0,27 & 0,08 & 0,32 & 0,68 & 0,35 \\
\hline 75. Cupania vernalis & 3 & 0,16 & 0,17 & 0,32 & 0,66 & 0,33 \\
\hline 76. Unonopsis lindmanii & 4 & 0,22 & 0,09 & 0,32 & 0,63 & 0,31 \\
\hline 77. Rheedia gardneriana & 4 & 0,22 & 0,08 & 0,32 & 0,62 & 0,30 \\
\hline 78. Machaerium sp. & 5 & 0,27 & 0,01 & 0,32 & 0,61 & 0,29 \\
\hline 79. Cassia sp. & 4 & 0,22 & 0,06 & 0,32 & 0,60 & 0,28 \\
\hline 80. Nectandra cissiflora & 2 & 0,11 & 0,22 & 0,21 & 0,54 & 0,33 \\
\hline 81. Aspidosperma cylindrocarpum & 3 & 0,11 & 0,19 & 0,21 & 0,51 & 0,30 \\
\hline 82. Machaerium nictitans & 3 & 0,16 & 0,01 & 0,32 & 0,50 & 0,18 \\
\hline 83. Eugenia florida & 3 & 0,16 & 0,01 & 0,32 & 0,50 & 0,17 \\
\hline 84. Myrcia sp.1 & 3 & 0,16 & 0,01 & 0,32 & 0,50 & 0,17 \\
\hline 85. Mouriri apiranga & 2 & 0,11 & 0,16 & 0,21 & 0,48 & 0,27 \\
\hline 86. Ormosia arborea & 2 & 0,11 & 0,15 & 0,21 & 0,47 & 0,26 \\
\hline 87. Agonandra brasiliensis & 2 & 0,11 & 0,20 & 0,11 & 0,41 & 0,31 \\
\hline 88. Maytenus sp.1 & 3 & 0,16 & 0,01 & 0,21 & 0,39 & 0,12 \\
\hline 89. Pera glabrata & 2 & 0,11 & 0,06 & 0,21 & 0,38 & 0,17 \\
\hline 90. Ficus cf. trigonata & 1 & 0,05 & 0,22 & 0,11 & 0,38 & 0,27 \\
\hline 91. Cassia ferruginea & 2 & 0,11 & 0,05 & 0,21 & 0,37 & 0,16 \\
\hline 92. Guapira cacerensis & 2 & 0,11 & 0,04 & 0,21 & 0,36 & 0,15 \\
\hline 93. Vochysia tucanorum & 1 & 0,05 & 0,18 & 0,11 & 0,34 & 0,23 \\
\hline 94. Gomidesia brunea & 2 & 0,11 & 0,01 & 0,21 & 0,33 & 0,12 \\
\hline 95. Bauhinia sp. & 2 & 0,11 & 0,01 & 0,21 & 0,33 & 0,12 \\
\hline 96. Sorocea bomplandii & 2 & 0,11 & 0,01 & 0,21 & 0,33 & 0,12 \\
\hline 97. Cedrela fissilis & 2 & 0,11 & 0,00 & 0,21 & 0,33 & 0,11 \\
\hline 98. Piper arboreum & 3 & 0,16 & 0,01 & 0,11 & 0,28 & 0,17 \\
\hline 99. Metrodorea pubescens & 1 & 0,05 & 0,08 & 0,11 & 0,24 & 0,14 \\
\hline 100 Zanthoxylum riedelianum & 2 & 0,11 & 0,01 & 0,11 & 0,23 & 0,12 \\
\hline 101. Prunus sp. & 1 & 0,05 & 0,05 & 0,11 & 0,21 & 0,10 \\
\hline 102. Celtis iguanae & 1 & 0,05 & 0,03 & 0,11 & 0,19 & 0,09 \\
\hline 103. Rapanea umbellata & 1 & 0,05 & 0,03 & 0,11 & 0,19 & 0,08 \\
\hline 104. Casearia gossypiosperma & 1 & 0,05 & 0,02 & 0,11 & 0,18 & 0,07 \\
\hline 105. Maclura tinctoria & 1 & 0,05 & 0,01 & 0,11 & 0,18 & 0,07 \\
\hline 106. Machaerium stipitatum & 1 & 0,05 & 0,01 & 0,11 & 0,18 & 0,07 \\
\hline 107. Calyptranthes clusiaefolia & 1 & 0,05 & 0,01 & 0,11 & 0,17 & 0,07 \\
\hline 108. Cardiopetalum calophyllum & 1 & 0,05 & 0,01 & 0,11 & 0,17 & 0,07 \\
\hline 109. Inga sp. 2 & 1 & 0,05 & 0,01 & 0,11 & 0,17 & 0,07 \\
\hline 110. Myrcia tomentosa & 1 & 0,05 & 0,01 & 0,11 & 0,17 & 0,06 \\
\hline 111. Myrcia sp. 2 & 1 & 0,05 & 0,00 & 0,11 & 0,16 & 0,06 \\
\hline 112. Xylopia aromatica & 1 & 0,05 & 0,00 & 0,11 & 0,16 & 0,06 \\
\hline 113. Psychotria sp. & 1 & 0,05 & 0,00 & 0,11 & 0,16 & 0,06 \\
\hline
\end{tabular}


IVI, nos dois ambientes (figura 1). Licania apetala ocorreu em ambas as situações, podendo ser uma espécie com características de secundária inicial, enquanto que Micrandra elata e Copaifera langsdorffii foram encontradas nas áreas de clareiras, principalmente como indivíduos adultos.

As espécies Alchornia glandulosa e Piptocarpha macropoda tiveram baixos valores de IVI na área intacta, ocorrendo nas clareiras com alta densidade de indivíduos jovens. Observadas em clareiras de vários tamanhos e na borda da mata, podem ser consideradas as principais espécies arbóreas pioneiras, do bosque de Araguari. Didymopanax morototoni, secundária inicial (Gandolfi et al. 1995) e Maprounea guianensis, tiveram baixos valores de IVI na área intacta, o que indica preferência pela área de clareira. Além dessas, Siparuna guianensis foi a única espécie do subosque que ocorreu entre as dez de maior IVI nas clareiras. Ocotea spixiana e Qualea jundiay foram encontradas no subosque e no dossel superior da mata fechada. Estas duas últimas espécies não são tipicamente de áreas abertas, podendo indicar diferentes estádios de clareiras no interior da mata do Bosque John Kennedy.

A comunidade vegetal do bosque de Araguari, é semelhante às reservas nativas da região, ou seja, possui áreas fechadas, com árvores altas e copas bem fechadas, ao lado de clareiras em formação e em fase de recuperação. Apesar de ser mata urbana, sujeita à ação antrópica, ainda mantém alta diversidade florística natural. Em vista do crescente fluxo de pessoas, que procuram essa área verde para caminhadas e turismo ecológico, é necessário um firme propósito de conservação, com plano adequado de manejo, visando à manutenção de suas características naturais.

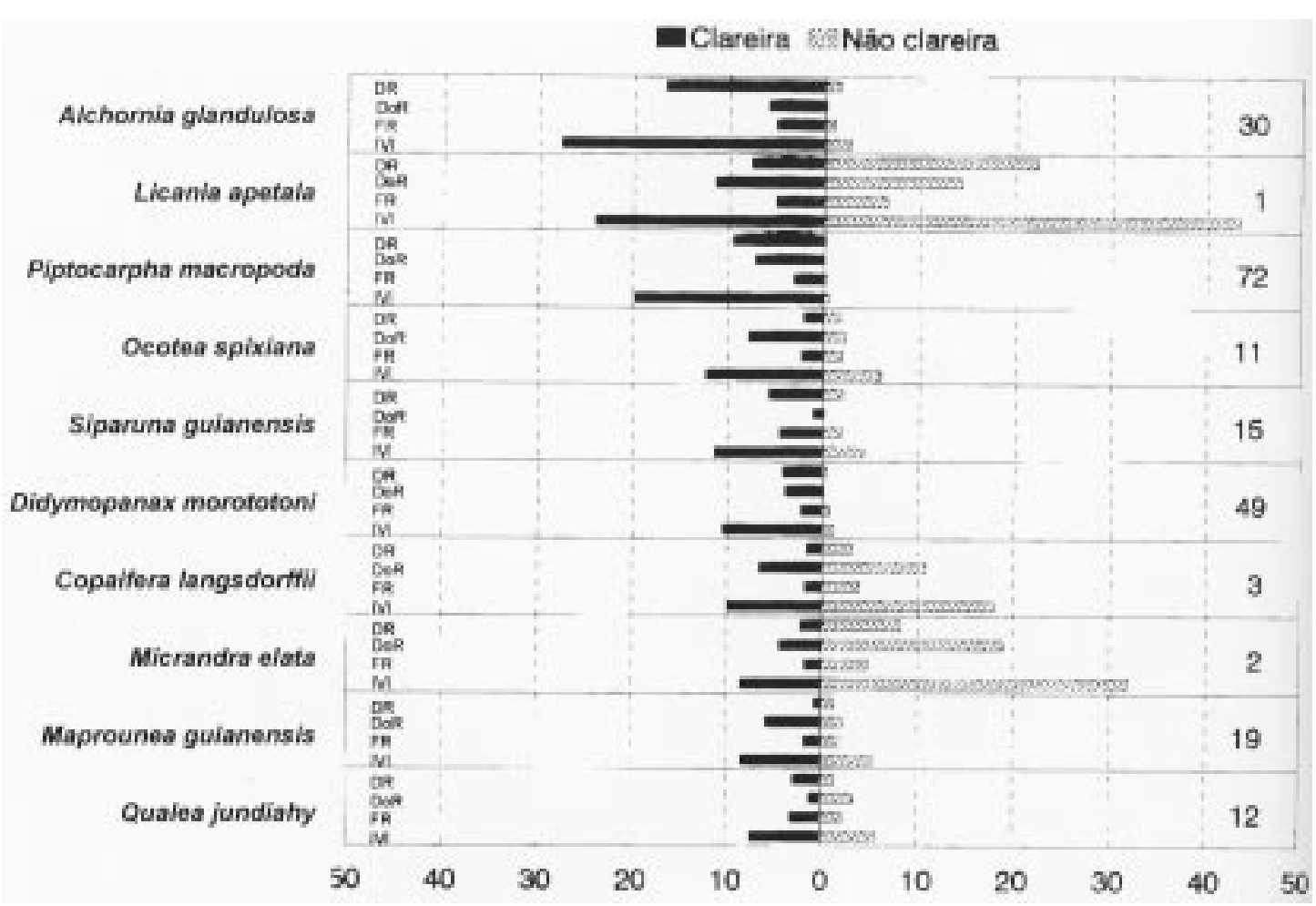

Figura 1. As dez principais espécies, em ordem de índice de valor de importância (IVI), encontradas nas clareiras da mata mesófila do bosque de Araguari, Minas Gerais, os outros parâmetros fitossociológicos estimados $(\mathrm{em} \%)$ : DR = densidade relativa, $(\mathrm{DoR})=$ dominância relativa e $(F R)=$ frequiência relativa, e os seus respectivos valores nas áreas fechadas (não clareiras). Os números no interior da figura representam a posição do IVI das espécies de áreas fechadas. 


\section{Referências bibliográficas}

ARAÚJO, G.M. 1992. Comparação da estrutura e do teor de nutrientes nos solos e nas folhas de espécies arbóreas de duas matas semidecíduas no Triângulo Mineiro. Tese de doutorado, Universidade Estadual de Campinas, Campinas.

BACCARO, C.A.D. 1990. Estudo dos processos geomorfológicos de escoamento pluvial em áras de cerrado Uberlândia, MG. Tese de doutorado, Universidade de São Paulo, São Paulo.

BERTONI, J.E.A. \& MARTINS, F.R. 1987. Composição florística de uma floresta ripária na Reserva Estadual de Porto Ferreira (SP). Acta Bot. Brasil. 1:17-26.

CAVASAN, O., CESAR, O. \& MARTINS, F.R. 1984. Fitossociologia da vegetação arbórea da Reserva Estadual de Bauru, estado de São Paulo. Revta brasil. Bot. 7:91-106.

CESAR, O. 1988. Composição florística, fitossociologia e ciclagem de nutrientes em mata mesófila semidecídua (Fazenda Barreiro Rico, Mun. Anhembi (SP). Tese de livre docência, Universidade Estadual de São Paulo, Rio Claro.

CFSMG. 1979. Recomendações para uso de corretivos e fertilizantes em Minas Gerais, 3ª aproximação, EPAMIG, Belo Horizonte.

DENSLOW, J.S. 1980. Gap partitioning among tropical rainforest succession trees. Biotropica 12:47-55

DENSLOW, J.S. 1987. Tropical rainforest gaps and tree species diversity. Annu. Rev. Ecol. Syst. 18:431-451.

ElTEN, G. 1990. Vegetação. In Cerrado: caracterização, ocupação e perspectivas (M.N. Pinto, org.). Edit. da Universidade de Brasília, Brasília, p.9-55.

EMBRAPA. 1979. Serviço nacional de levantamento e conservação dos solos. Manual de métodos de análise do solo, Rio de Janeiro.

EMBRAPA. 1982. Serviço nacional de levantamento e conservação dos solos. Levantamento de média intensidade dos solos e a avaliação da aptidão agrícola das terras do Triângulo Mineiro. Bol. de Pesquisa 1, Rio de Janeiro.

FELFILI, J.M. \& SILVA JR, M.C. 1992. Floristic composition, phytosociology and comparison of cerrado and gallery forests at Fazenda Água Limpa, Federal District, Brazil. In Nature and dynamics of forest-savanna boundaries (P. A. Furley, J. Proctor \& J. A. Ratter, eds.). Chapman \& Hall, London, p.393-415.

GANDOLFI, S., LEITÃO-FILHO, H.F. \& BEZERRA, C.L. 1995. Levantamento florístico e caráter sucessional das espécies arbustivo-arbóreas de uma floresta mesófila semidecídua no Município de Guarulhos, SP. Revta brasil. Biol. 55:753-767.

GREIG-SMITH, C.T. 1983. Quantitative plant ecology. University of California Press, Los Angeles.

LEITÃO-FILHO, H.F. 1986. Considerações sobre a florística de florestas tropicais e sub-tropicais do Brasil. In Anais de Mesa redonda sobre a conservação "in situ” de florestas tropicais. Piracicaba, São Paulo, p.1-26.

MARTINS, F.R. 1991. Estrutura de uma floresta mesófila. Editora da Universidade Estadual de Campinas, Campinas.

MATHES, L.A.F., LEITÃO FILHO, H.E. \& MARTINS, F.R. 1988. Bosque dos jequitibás (Campinas, SP): composição florística e estrutura fitossociológica do estrato arbóreo. In Anais V Congresso da SBSP. p.55-76.

MEIRA-NETO, J.A.A., BERNACCI, L.C., GROMBONE, M.T., TAMASHIRO, J.Y. \& LEITÃO-FILHO, H.F. 1989. Composição florística da floresta semidecídua de altitude do Parque Municipal da Grota Funda (Atibaia, estado de São Paulo). Acta Bot. Brasil. 3:51-74.

MUELLER-DOMBOIS, D. \& ELLEMBERG, H. 1974. Aims and methods of vegetation ecology. John Wiley \& Sons, New York.

OLIVEIRA-FILHO, A.T., SCOLFORO, J.R.S. \& MELLO, J.M. 1994. Composição florística e estrutura comunitária de um remanescente de floresta semidecídua montana em Lavras, MG. Revta brasil. Bot. 17:167-182

PAGANO, S.N., LEITÃO-FILHO, H.F. \& SHEPHERD, G.J. 1987. Estudo fitossociológico em mata mesófila semidecídua no município de Rio Claro, (Estado de São Paulo). Revta brasil. Bot. 10:49-61.

RATTER, J.A., ASKEW, G.P., MONTGOMERY, R.F. \& GIFFORD, D.R. 1978a. Observation on the vegetation of northeastern Mato Grosso II. Forest and soil of the Rio Suiá-Missu area. Proc. R. Soc. Lond. 203:191-208.

RATTER, J.A., FURLEY, P.A., MONTGOMERY, R.F. \& GIFFORD, D.R. 1978b. Observations on forests of some mesotrophic soils in Central Brazil. Revta brasil. Bot. $1: 47-58$

RIZZINI, C.T. 1963. Nota prévia sobre a divisão fitogeográfica (florístico-sociológica) do Brasil. Revta brasil. Geogr. 1:3-55.

RIZZINI, C. T. 1979. Tratado de fitogeografia do Brasil, aspectos sociológicos e florísticos v.2. Editora da Universidade de São Paulo, São Paulo.

RODRIGUES, R.R. 1986. Levantamento florístico e fitossociológico das matas da Serra do Japi, Jundiaí, SP. Tese de mestrado, Universidade Estadual de Campinas, Campinas.

RODRIGUES, R.R., MORELLATO, L.P.C., JOLY, C.A. \& LEITÃO-FILHO, H.F. 1989. Estudo florístico e fitossociológico em um gradiente altitudinal de mata estacional mesófila semidecídua, na Serra do Japi, Jundiaí, SP. Revta brasil. Bot. 12:71-84.

SANTIN, D .A. 1991. - Astronium nelson-rosae - Nova espécie de Anacardiaceae. Revta brasil. Bot. 12:103-106.

SCHIAVINI, I. 1992. Estrutura das comunidades arbóreas de mata de galeria da Estação Ecológica do Panga (Uberlândia, MG). Tese de doutorado, Universidade Estadual de Campinas, Campinas. 